\title{
The existence of steady states to a combustion model with internal heating
}

\author{
A.A. Shah ${ }^{\mathrm{a}, *}$, G.C. Wake ${ }^{\mathrm{b}}$ \\ a Applied Mathematics Department and Department of Fuel and Energy, University of Leeds, \\ Leeds LS2 9JT, West Yorkshire, UK \\ ${ }^{\mathrm{b}}$ Department of Mathematics and Statistics, University of Canterbury, Private Bag 4800, \\ Christchurch, New Zealand
}

Received 14 March 2003; accepted 11 November 2003

Keywords: Combustion; Steady states; Existence; Activation-energy asymptotics

\section{Introduction}

In recent numerical studies [5-7,20,26], the authors examined the effect of a constant source of heat placed at the centre of a reactive solid $X$. Reactions with and without oxygen, in all class-A geometries (slab, concentric cyclinders, concentric spheres) were considered. The background and the motivation for these numerical studies was the thermal decomposition of combustible solids such a bulk powders and coal dust, occurring for example in many operations in the process control industry. Equipment such as blenders, mills and screw feeders can develop into localized sources of heat from mechanical impact or failure of a bearing. This source of heat, or 'hot-spot', can be sufficient to cause slow local combustion of the surrounding material, or even initiate a self-propagating combustion wave. Clearly an understanding of the conditions under which thermal decomposition occurs and the extent of the decomposition are crucial for hazard prevention. In this work we study the existence and nonexistence of steady states to the model in [5], generalized to include the combustion of gases and non class-A shapes. That is, we imagine a gas or a solid containing a source of heat placed arbitrarily in its interior-independent of the heat produced by chemical reaction (see Fig. 1).

\footnotetext{
* Corresponding author. Tel.: +44-113-343-2392; fax: +44-113-244-3923.

E-mail address: preaas@sun.leeds.ac.uk (A.A. Shah).
} 


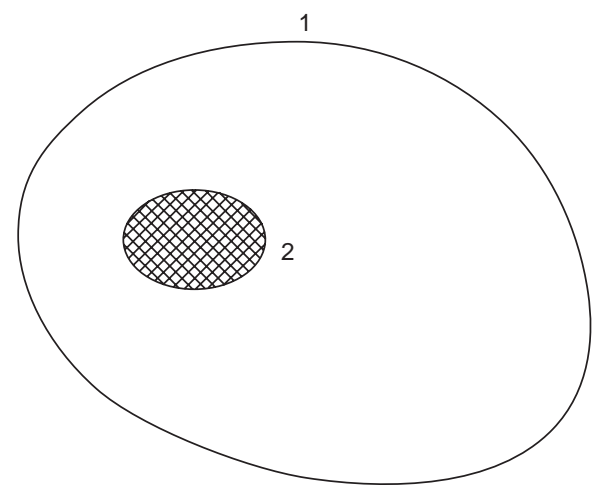

Fig. 1. A sketch of the configuration, showing the outer boundary $\Gamma_{1}$ (or $\Gamma_{1}^{\prime}$ ) and the inner boundary $\Gamma_{2}$ ( or $\Gamma_{2}^{\prime}$ ), which coincides with the surface of the heating source ("hot-spot"). The latter is represented by the shaded region.

Similar numerical studies [5-7,20], have been carried out in, for example, [15,27], where the authors focus on predicting the critical initial conditions for the safe storage of potentially exothermic materials; a study of spontaneous ignition. On the other hand, the character and properties of a fully developed combustion wave have been addressed in, amongst others, [14,22,23]. Analytical progress in describing the ignition event using the equations in [5] goes back to Liñán and Williams [19], and Kapila [16], in which the authors develop an asymptotic theory for the ignition of a solid slab subjected to a constant heat flux. The former work neglects reactant consumption and in the latter the author only addresses the gaseous problem with equidiffusion (of heat and mass).

The purpose of the present study lies in the relationship between the steady-state solutions and the critical behaviour of the original system. This is most easily demonstrated by considering a single equation with Dirichlet data, describing the evolution of temperature and which can be considered as an eigenvalue problem. If one uses the Frank-Kamenetskii approximation (reaction rate $\propto \mathrm{e}^{u}$, where $u$ is temperature), it is well known that the spectrum of the steady-state problem is bounded above. Furthermore, for a reaction-rate coefficient smaller than the upper bound, i.e. when a steady-state exists, the solution relaxes to one of the steady states, provided the initial condition is small enough. For values greater than the upper bound the solution can become infinite in finite time; a situation referred to as blow-up. Moreover, a study regarding reactant-dependent equations has been performed in [9-11], yielding results of a similar nature. For further details see [9] and references therein. These ideas will be made more precise in the context of our problem in a future publication, in which we examine the behaviour (including probable multiplicity) of steady-state solutions as various parameters are varied. Here we concentrate solely on the question of the existence of at least one steady state.

In this work we use a reaction-rate of the type $\mathrm{e}^{-1 / u}$, corresponding to the nondimensionalization used in [5,6] and to the full ignition problem for both gases and solids. We show that steady-state solutions exist for all Damköhler numbers - a measure of 
the rapidity of the reaction (see [8] for details). The Frank-Kamenetskii approximation [12] in this model of the burning corresponds to a perturbation problem about a reference temperature and concentration, and is derived under certain asymptotic-limit assumptions and by assuming that the power of the heat source is asymptotically small. This situation is entirely realistic for bulk materials such as compost, moist wood chips, lead azide and ammonium nitrate, where the reactant consumption is very small. We are able to demonstrate that steady-state solutions to the perturbation problem do not exist for all finite Damköhler numbers and are able to find an upper limit of Damköhler number (above which no solution exists).

In the next section we provide some definitions necessary for the work that follows. The mathematical problem is stated in Section 3 and in Section 4 we lay the foundation for the analysis. Based on maximum and comparison principles, in Section 5 we prove that steady-state solutions of the full problem lie in an invariant set, with respect to boundary conditions. The proof of existence for the full problem then appears in Section 6. The remaining part of the paper is devoted to the perturbation problem in which the Frank-Kamenetskii approximation is used.

\section{Preliminaries and notation}

$C_{\alpha}(\Omega)$ as the space of Hölder continuous functions in $\Omega \subset \mathbb{R}^{n}$, with exponent $0 \leqslant \alpha \leqslant 1$. A norm on these spaces is defined by

$$
|f|_{C_{\alpha}(\Omega)}=|f|_{\alpha}^{\Omega}:=\sup _{\Omega}|f|+[f]_{\alpha}^{\Omega},
$$

where $[f]_{\alpha}^{\Omega}:=\sup _{x, y \in \Omega}|f(x)-f(y)| /|x-y|^{\alpha}$. We shall include the space of continuous functions $C(\Omega)$ among the $C_{\alpha}(\Omega)$ as those corresponding to $\alpha=0 . C_{\alpha, k}(\Omega)$ consists of functions whose partial derivatives up to and including order $k$ have a finite $|\cdot|_{\alpha}^{\Omega}$ norm, so a norm on these spaces is given by

$$
|f|_{C_{\alpha, k}(\Omega)}=|f|_{k, \alpha}^{\Omega}:=\sum_{j=0}^{k} \sup _{|\beta|=j} \sup _{\Omega}\left|D^{\beta} f\right|+\sup _{|\beta|=k}\left[D^{\beta} u\right]_{\alpha}^{\Omega}
$$

for multi-index $\beta$. An equivalent and more convenient norm which we will use for estimates up to the boundary is given by

$$
|f|_{k, \alpha}^{\Omega *}:=\sum_{j=0}^{k} d^{j} \sup _{|\beta|=j} \sup _{\Omega}\left|D^{\beta} f\right|+d^{k+\alpha} \sup _{|\beta|=k}\left[D^{\beta} u\right]_{\alpha}^{\Omega},
$$

where $d=\operatorname{diam}(\Omega)$. In the case of vector valued functions $F: \Omega \rightarrow \mathbb{R}^{m}$, we define a norm on the space $C_{\alpha, k}(\Omega)^{m}:=\prod_{i=1}^{m} C_{\alpha, k}(\Omega)$ by $|F|_{C_{\alpha, k}(\Omega)^{m}}:=\prod_{i=1}^{m}\left|F_{i}\right|_{C_{\alpha, k}(\Omega)}$, and we can similarly define $|\cdot|^{*}$ norms.

The space $L^{p}(\Omega)$ is the Banach space of $p$ th Lebesgue integrable equivalence-classes of functions endowed with the norm $\|f\|_{L^{p}(\Omega)}=\|f\|_{p}^{\Omega}:=\left\{\int_{\Omega}|f|^{p}\right\}^{1 / p}$ for $1 \leqslant p \leqslant \infty$. In the case $p=2$ we shall omit the subscript on the norm and the associated inner

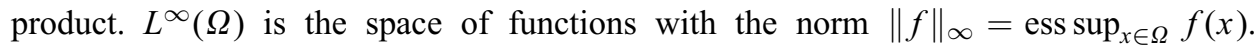


$W^{k, p}(\Omega), k, p \in \mathbb{N}$, is the Sobolev space of functions with norm $\|f\|_{W^{k, p}(\Omega)}=\|f\|_{k, p}^{\Omega}:=$ $\left\{\int_{\Omega} \sum_{|j| \leqslant k}\left|D^{j} f\right|^{p}\right\}^{1 / p}$, where the derivatives $D^{k} f$ are understood to exist in the weak sense and $j$ is a multi-index. In this and the previous norm definition, we shall omit the superscript $\Omega$ if it is clear from the context to which region or manifold we refer. In the case of vector valued functions $F: \Omega \rightarrow \mathbb{R}^{m}$, we define a norm on the space $W^{k, p}(\Omega)^{m}:=\prod_{i=1}^{m} W^{k, p}(\Omega)$ by $\|F\|_{W^{k, p}(\Omega)^{m}}:=\prod_{i=1}^{m}\left\|F_{i}\right\|_{W^{k, p}(\Omega)}$. When $p=2$ and $k>0$ is a noninteger, we write $k=K+l$, where $K \in \mathbb{N}$ and $0<l<1$, and define the Sobolev-Slobodeckii inner product

$$
(f, g)_{W^{k, 2}(\Omega)}:=(f, g)_{W^{K, 2}(\Omega)}+\sum_{|j|=K} \int_{\Omega \times \Omega} \frac{\left(D^{j} f(x)-D^{j} f(y)\right)\left(D^{j} g(x)-D^{j} g(y)\right)}{|x-y|^{n+2 \lambda}},
$$

which is used to define a norm $\|f\|_{W^{k, 2}(\Omega)}:=(f, f)_{W^{k, 2}(\Omega)}^{1 / 2}$ on these spaces. A more precise definition and details can be found in [28]. For more details concerning Hölder continuity see [13]. The notation $X \underset{\text { comp }}{\rightarrow} Y$ will be used for the compact imbedding of a space $X$ in a space $Y$ and $C^{m}(\Omega)$ is the space of scalar-valued functions on a bounded domain $\Omega$, whose derivatives up to and including order $m$ are bounded-continuous functions.

The symbol $\varnothing$ is used for a zero vector.

Finally, we define the following partial orderings for members of $\mathbb{R}^{n}$ : Let $a, b \in \mathbb{R}^{n}$; we write $a \prec b$ if $a_{i}<b_{i}, \forall i=1, \ldots, n$. Similarly we define $a \succ b, a \preccurlyeq b$ and $a \succcurlyeq b$.

\section{Mathematical model}

For simplicity, and as in [5], we assume that the fully burnt material occupies the same volume and has the same characteristics as the unburnt material and undergoes reaction according to the Arrhenius law. The former assumption is quite valid for reactions that do not involve a change of phase, and the latter assumption is common in combustion modelling. A general form of the equations representing temperature $T$ and reactant mass fraction $X$ in a combustion process is as follows:

$$
\left.\begin{array}{c}
\rho C_{p} \frac{\partial T}{\partial t^{\prime}}-\kappa \nabla^{\prime 2} T=Q^{\prime} A X \mathrm{e}^{-E_{\mathrm{a}} / R T} \\
\rho\left(\frac{\partial X}{\partial t^{\prime}}-D_{X} \nabla^{\prime 2} X\right)=-A X \mathrm{e}^{-E_{\mathrm{a}} / R T}
\end{array}\right\} \quad \text { on } \Omega^{\prime}
$$

in which $E_{\mathrm{a}}$ is the activation energy, $\rho$ is the density of the reactant, $C_{p}$ is its specific heat capacity, $D_{X}$ its diffusivity, $\kappa$ is its thermal conductivity, $A$ is the pre-exponential constant and $R$ is the universal gas constant. $Q$ is the heat-release of the reaction and the reactant occupies the bounded region $\Omega^{\prime} \subset \mathbb{R}, n \in\{2,3\}$, between the surface of the heat source $\Gamma_{2}^{\prime}$ and the outer edge of the reactive material $\Gamma_{1}^{\prime}$ (see Fig. 1). In the case of a gaseous reactant the gas-dynamic equations are decoupled from these equations by assuming that the reactant is a small fraction of a mostly inert mixture, with density, specific heat capacity and thermal conductivity equal to $\rho, C_{p}$ and $\kappa$ respectively. The latter are constant to a good approximation when one assumes that 
the chemical heat-release is small compared to the internal energy of the mixture-the so-called weak heat-release approximation.

On $\Gamma_{1}^{\prime}$ the reactant remains unreacted at the ambient temperature $T_{0}$ and at the inner boundary $\Gamma_{2}^{\prime}$ a heat flux $\tilde{P}$ emanates from the hot-spot. The boundary and initial conditions that accompany Eqs. (1) are therefore

$$
\begin{aligned}
& T\left(x^{\prime}, 0\right)=\hat{T}_{0}\left(x^{\prime}\right), \quad X\left(x^{\prime}, 0\right)=\hat{X}_{0}\left(x^{\prime}\right), \quad T=T_{0}\left(x^{\prime}\right), \quad X=X_{0}\left(x^{\prime}\right) \quad \text { on } \Gamma_{1}^{\prime}, \\
& \kappa \frac{\partial T}{\partial n^{\prime}}=\tilde{P}\left(x^{\prime}\right), \quad \frac{\partial X}{\partial n^{\prime}}+\beta^{\prime}\left(x^{\prime}\right) X=0 \quad \text { on } \Gamma_{2}^{\prime},
\end{aligned}
$$

where $n$ denotes the normal pointing outward from $\partial \Omega^{\prime}=\Gamma_{1}^{\prime} \cup \Gamma_{2}^{\prime}$. We assume that $\hat{T}_{0}$ and $T_{0}$, and $\hat{X}_{0}$ and $X_{0}$ satisfy the compatibility conditions

$$
\hat{T}_{0}=T_{0}, \quad \hat{X}_{0}=X_{0} \quad \text { on } \Gamma_{1}^{\prime} .
$$

It is also assumed that $\hat{T}_{0}$ is everywhere below the temperature required for instantaneous combustion and $\hat{X}_{0}>0$. It is far more convenient to work with dimensionless variables and so we rescale as follows:

$$
\begin{aligned}
& x=\frac{x^{\prime}}{\left|x^{\prime}\right|}, \quad t=\frac{\kappa t^{\prime}}{\rho C_{p}\left|x_{1}^{\prime}\right|^{2}}, \quad u=\frac{R T}{E_{\mathrm{a}}}, \quad v=\frac{X}{\left|\hat{X}_{0}\right|_{C(\Omega)}}, \quad \varepsilon=\frac{\rho C_{p} D_{X}\left|x_{1}^{\prime}\right|^{2}}{\kappa}, \\
& Q=\frac{Q^{\prime} R}{C_{p} E_{\mathrm{a}}}, \quad \alpha=\frac{\tilde{P} R\left|x_{1}^{\prime}\right|}{\kappa E_{\mathrm{a}}}, \quad \mathscr{D}=\frac{A \rho C_{p}\left|x_{1}^{\prime}\right|^{2}}{\kappa}, \quad u_{0}=\frac{R T_{0}}{E_{\mathrm{a}}}, \quad v_{0}=\frac{X_{0}}{\left|\hat{X}_{0}\right| C(\Omega)}, \\
& \hat{u}_{0}=\frac{R \hat{T}_{0}}{E_{\mathrm{a}}}, \quad \hat{v}_{0}=\frac{\hat{X}_{0}}{\left|\hat{X}_{0}\right|_{C(\Omega)}}, \quad \beta(x)=\frac{\left|\hat{X}_{0}\right| C(\Omega)}{\left|x_{1}^{\prime}\right|} \beta^{\prime}\left(x\left|x_{1}^{\prime}\right|\right), \\
& \Omega^{\prime} \mapsto \Omega, \quad \Gamma_{1}^{\prime} \mapsto \Gamma_{1}, \quad \Gamma_{2}^{\prime} \mapsto \Gamma_{2},
\end{aligned}
$$

where $\left|x_{1}^{\prime}\right|=\operatorname{diam}(\Omega) / 2$. Substituting (3) into Eqs. (1) and (2) now yields

$$
\left.\begin{array}{ll}
\frac{\partial u}{\partial t}-\nabla^{2} u & =Q \mathscr{D} v \mathrm{e}^{-1 / u} \\
\frac{\partial v}{\partial t}-\varepsilon \nabla^{2} v & =-\mathscr{D} v \mathrm{e}^{-1 / u}
\end{array}\right\} \quad \text { on } \Omega
$$

and

$$
\begin{aligned}
& u(x, 0)=\hat{u}_{0}(x), \quad v(x, 0)=\hat{v}_{0}(x), \quad u=u_{0}(x), \quad v=v_{0}(x) \quad \text { on } \Gamma_{1}, \\
& \frac{\partial u}{\partial n}=\alpha(x), \quad \frac{\partial v}{\partial n}+\beta(x) v=0 \quad \text { on } \Gamma_{2} .
\end{aligned}
$$

In equation (3), $\mathscr{D}$ is the Damköhler number, which is a measure of the rapidity of the reaction, and $\varepsilon>0$ is the Lewis number, measuring the rate of transport of heat relative to the rate of transport of the material. For gases $\varepsilon \approx 1$, whereas the solid limit is $\varepsilon \rightarrow \infty$.

At this point we replace $\mathscr{D} \mathrm{e}^{-1 / u}$ by a more general function $\omega(u)$. The reason for this is that it will allow an easier comparison with previous works of this nature (see for example [4]). In addition, the perturbation problem we consider in Section 7 has 
an entirely different reaction rate, and so we draw the distinction now. The following are the main assumptions on the boundary, the reaction rates $\omega(u)$ and the coefficients:

(BA) $\Omega$ is doubly-connected with an outer boundary $\Gamma_{1}$ and inner boundary $\Gamma_{2}$ satisfying $\partial \Omega=\Gamma_{1} \cup \Gamma_{2}, \Gamma_{1} \cap \Gamma_{2}=\emptyset$, and where both enclose simply-connected regions in $\mathbb{R}^{n}$ (see Fig. 1). We shall assume that $\Omega$ satisfies at least the uniform cone (and therefore segment) property, by the choices $\Gamma_{1}, \Gamma_{2} \in C^{2,1}$.

(RA) $\omega$ is either:

- Type I: Hölder continuous in $u$ with exponent $b$, and is bounded both above and below:

$$
\begin{aligned}
& \omega(u) \in C_{b}\left(\mathbb{R}_{+}\right), \quad 0 \leqslant \omega(u) \leqslant \omega_{u} \forall u \in \mathbb{R}_{+}, \\
& \lim \inf _{s \rightarrow \infty} \frac{\omega(s)}{s}=0 \quad \text { uniformly. }
\end{aligned}
$$

The latter condition demands sublinear growth as $s \rightarrow \infty$. These assumptions are consistent with a function of the type $\mathrm{e}^{-1 / u}$, which corresponds to the full ignition problem for temperature and mass fraction.

- Type II: $\omega \in C^{1}(\mathbb{R})$ and satisfies

$$
\omega(s)>0 \quad \forall s \in \mathbb{R}_{+}, \quad \lim \inf _{s \rightarrow \infty} \frac{\omega(s)}{s}>0 \quad \text { uniformly }
$$

and there exist nonnegative constants $k_{1}$ and $k_{2}$ such that

$$
\omega(u) \geqslant k_{1}+k_{2} u \text {. }
$$

The function $\mathrm{e}^{u}$ satisfies these conditions and corresponds to a problem in which $u$ is a temperature perturbation, of the order of the inverse of the activation energy, about its ambient value, and $\alpha$ is of the same order of magnitude. In this case the equation for the corresponding perturbation in mass fraction is decoupled from the temperature equation at leading order. The relevance of this problem was outlined in the introduction and will be reiterated in Section 7.

(CA) The boundary terms satisfy $u_{0}, v_{0}>0$ and

$$
\begin{gathered}
u_{0}, v_{0} \in C_{2, b}\left(\Gamma_{1}\right) \cap W^{\frac{3}{2}, 2}\left(\Gamma_{1}\right), \quad \alpha \in C_{1, b}\left(\Gamma_{2}\right) \cap W^{\frac{1}{2}, 2}\left(\Gamma_{2}\right), \\
\beta \in W^{1, \infty}\left(\Gamma_{2}\right) \cap C_{1, b}\left(\Gamma_{2}\right)
\end{gathered}
$$

with $\alpha, \beta>0$.

The steady states (using the same symbols) are found by neglecting the time variations in Eqs. (4) and (5) (after replacing $\mathrm{e}^{-1 / u}$ with $\omega(u)$ ):

$$
\left.\begin{array}{l}
\nabla^{2} u+Q v \omega(u)=0 \\
-\varepsilon \nabla^{2} v+v \omega(u)=0
\end{array}\right\} \quad \text { on } \Omega,
$$

together with the boundary conditions

$$
\begin{aligned}
& u=u_{0}(x), \quad v=v_{0}(x) \quad \text { on } \Gamma_{1} \\
& \frac{\partial u}{\partial n}=\alpha(x), \quad \frac{\partial v}{\partial n}+\beta(x) v=0 \quad \text { on } \Gamma_{2} .
\end{aligned}
$$




\section{Fixed points of the abstract problem}

The existence proofs are based on locating fixed points of a completely continuous (relatively compact and continuous) map using Leray-Schauder degree $\operatorname{deg}(a, b, c)$, an integer measuring in a continuous fashion the number of zeros of the map. Suppose that we seek the Leray-Schauder degree of the equality $f(x)=y$, where $f: E \supset \Sigma \rightarrow E$ and $y$ is a point in the range of $f$. Then $a, b$ and $c$ in the symbol above would respectively represent the map $f$, its domain $\Sigma$ and the point $y$. The results from degree theory that will be relevant to our existence proofs are given in the following lemma.

Lemma 4.1 (Homotopy invariance). Let $f_{\lambda}=I-F(x, \lambda)$, where $I$ is the identity map and $F: \bar{\Sigma} \times[0,1] \rightarrow E$ is completely continuous with $F(x, 0)=\varnothing$. Suppose that $\varnothing \notin \partial \Sigma$ for all $\lambda \in[0,1]$. Then $\operatorname{deg}\left(f_{\lambda}, \Sigma, \varnothing\right)$ is defined, is independent of $\lambda$ and $\operatorname{deg}\left(f_{0}, \Sigma, \varnothing\right)=1$.

For a proof see [25, Theorem 5.3].

We now relate BVP (9)-(10) to an abstract problem in an appropriate function space $\mathscr{S}$ (selected later) which will allow us to associate it to a suitable homotopy. This in turn will allow us to employ Lemma 4.1 to locate a fixed point of the abstract problem, which by design will also be a solution to BVP (9)-(10). In what follows we shall assume that $n=3$. The extension of the results to $n<3$ is trivial. Let

$$
\underset{\sim}{u}:=(u, v)^{\mathrm{T}} \in \mathscr{S} \times \mathscr{S} \text { and } \quad \underset{\sim}{\hat{u}}=(\hat{u}, \hat{v})^{\mathrm{T}} \in \mathscr{S} \times \mathscr{S}
$$

supposing the latter is known, and consider the system

$$
\begin{aligned}
& E \underset{\sim}{\underset{\sim}{u}}(x)=R \underset{\sim}{\hat{u}}=\varnothing \quad \text { on } \Omega \\
& B \underset{\sim}{u}=G_{2} \quad \text { on } \Gamma_{1}, \quad I_{2} \underset{\sim}{u}=G_{1} \quad \text { on } \Gamma_{2},
\end{aligned}
$$

where the $2 \times 2$ matrix operators $E$ and $B$ are given by

$$
E_{i j}=\left\{\begin{array}{ll}
\nabla^{2}, & i=j=1, \\
-\varepsilon \nabla^{2}, & i=j=2, \\
0, & i \neq j,
\end{array} \quad B_{i j}= \begin{cases}\frac{\partial}{\partial n}, & i=j=1, \\
\frac{\partial}{\partial n}+\beta, & i=j=2, \\
0, & i \neq j .\end{cases}\right.
$$

$G_{1}=\left(u_{0}, v_{0}\right), G_{2}=(\alpha, 0)$ and $I_{2}$ is the $2 \times 2$ identity matrix. $R: \mathscr{S} \times \mathscr{S} \rightarrow \mathscr{S} \times \mathscr{S}$ is the Nemystkii operator

$$
R(\underset{\sim}{\hat{u}})(x):=\hat{v} \omega(\hat{u})(Q,-1)^{\mathrm{T}} .
$$

Now define the mapping $K: \mathscr{S} \times \mathscr{S} \rightarrow \mathscr{S} \times \mathscr{S}$ by $K \hat{\sim}=\underset{\sim}{u}$, i.e. $K \hat{\sim} \in \mathscr{S} \times \mathscr{S}$ is a solution to the linear and uncoupled system (11). Thus, we have reduced our original problem to that of finding a fixed point in $\mathscr{S} \times \mathscr{S}$ (or a subset of $\mathscr{S} \times \mathscr{S}$ ) of the operator $I-K$, where $I: \mathscr{S} \times \mathscr{S} \rightarrow \mathscr{S} \times \mathscr{S}$ is the identity mapping. Similar to the proofs in [4,9] we now construct a homotopy between $I$ and $I-K$ which will satisfy enough constraints to render Lemma 4.1 applicable. Let the solution operator $K$ of 
the linear problem be completely continuous. Further, let $K$ be such that: $\underset{\sim}{u}=\lambda K \underset{\sim}{u}$, $\lambda \in[0,1]$, if and only if it is a solution of the problem

$$
\left.\begin{array}{l}
\nabla^{2} u+Q \lambda v \omega(u)=0 \\
-\varepsilon \nabla^{2} v+\lambda v \omega(u)=0
\end{array}\right\} \quad \text { on } \Omega,
$$

together with the boundary conditions

$$
\begin{aligned}
& u=\lambda u_{0}(x), \quad v=\lambda v_{0}(x) \quad \text { on } \Gamma_{1}, \\
& \frac{\partial u}{\partial n}=\lambda \alpha(x), \quad \frac{\partial v}{\partial n}+\beta v=0 \quad \text { on } \Gamma_{2} .
\end{aligned}
$$

Then our homotopy $H: \mathscr{S} \times \mathscr{S} \rightarrow \mathscr{S} \times \mathscr{S}$ is defined by

$$
H(\underset{\sim}{u}, \lambda):=(I-\lambda K) \underset{\sim}{u}, \quad \lambda \in[0,1] .
$$

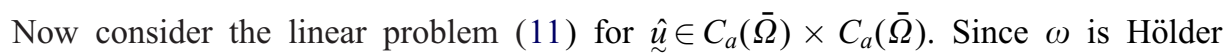
continuous (exponent $b$ ), $R: C_{a}(\bar{\Omega}) \times C_{a}(\bar{\Omega}) \rightarrow C_{a b}(\bar{\Omega}) \times C_{a b}(\bar{\Omega})$ is bounded for each $a \in[0,1]$ and continuous for $a=0$. The existence of solutions of (11) and their regularity follow from standard linear theory and these solutions satisfy the bounds

$$
|\underset{\sim}{\sim}|_{C_{2, a b}(\bar{\Omega})^{2}}^{*} \preccurlyeq C\left(|R \hat{\sim}|_{C_{a b}(\bar{\Omega})^{2}}+\left|G_{1}\right|_{C_{2, a b}\left(\Gamma_{1}\right)^{2}}+\left|G_{2}\right|_{C_{1, a b}\left(\Gamma_{2}\right)^{2}}\right)
$$

for $a \in(0,1]$. Thus we can define an operator $K: C_{a}(\bar{\Omega}) \times C_{a}(\bar{\Omega}) \rightarrow C_{2, a b}(\bar{\Omega}) \times C_{2, a b}(\bar{\Omega})$ by

$$
K \underset{\sim}{\hat{u}}=\underset{\sim}{u}
$$

and it satisfies the bound

$$
|K \underset{\sim}{\hat{\mathcal{u}}}|_{C_{2, a b}(\bar{\Omega})^{2}} \preccurlyeq C\left(|\hat{\sim}|_{C_{a}(\bar{\Omega})^{2}}+\left|G_{1}\right|_{C_{2, b}\left(\Gamma_{1}\right)^{2}}+\left|G_{2}\right|_{C_{1, b}\left(\Gamma_{2}\right)^{2}}\right) .
$$

We shall later appeal to a variational approach to show that $K$ has an extension (also labelled $K$ ) which is completely continuous. Ultimately, we need an extension $K: C(\bar{\Omega}) \times C(\bar{\Omega}) \rightarrow C(\bar{\Omega}) \times C(\bar{\Omega})$, which will define the $K$ in $(15)$.

Remark 4.1. Estimate (16) is a result of standard theory but is rarely stated explicitly in the literature. For the sake of completeness we present a sketch of the proof in the Appendix. See [17,18], particularly the introduction, for a discussion of related BVPs.

\section{A priori estimates}

We shall need estimates for the classical solutions of problem (13) and (14) to ensure that the Leray-Schauder degree is defined-in the sense that $\varnothing \notin \partial \Sigma_{\lambda}$ for $\lambda \in[0,1]$. For this we require the following result: 
Theorem 5.1. Let $u, v \in C^{2}(\Omega) \cap C(\bar{\Omega})$ be a solution of (9)-(10). Then

$$
0 \leqslant v \leqslant \bar{v} \quad \text { and } \quad \underline{u} \leqslant u \leqslant \bar{u},
$$

where $\bar{v}=\sup _{x \in \Gamma_{1}} v_{0}(x), \underline{u}=\inf _{x \in \Gamma_{1}} u_{0}(x)$ and $\bar{u}$ is a solution of the problem

$$
\nabla^{2} \bar{u}=-Q \omega_{u} \bar{v}<\infty \quad \text { on } \Omega, \quad \frac{\partial \bar{u}}{\partial n}=\alpha>0 \quad \text { on } \Gamma_{2}, \quad \bar{u}=u_{0} \quad \text { on } \Gamma_{1} .
$$

Theorem 5.1 is a consequence of classical maximum principles and Lemma 5.1 below, which relates to the mixed boundary value problem

$$
\left(\nabla^{2}+h(x)\right) z=f(x) \quad \text { on } \Omega, z=z_{0}(x) \text { on } \Gamma_{1}, \quad \frac{\partial z}{\partial n}+\beta z=g \quad \text { on } \Gamma_{2} .
$$

A lower (upper) solution $z_{l}(z u)$ of problem (21) is defined as

$$
\begin{aligned}
& \left(\nabla^{2}+h\right) z_{l} \geqslant f \quad \text { on } \Omega, \quad z_{l} \leqslant z_{0}(x) \quad \text { on } \Gamma_{1}, \quad \frac{\partial z_{l}}{\partial n}+\beta z_{l} \leqslant g \quad \text { on } \Gamma_{2} \text {, } \\
& \left(\nabla^{2}+h\right) z_{u} \leqslant f \quad \text { on } \Omega, \quad z_{u} \geqslant z_{0}(x) \quad \text { on } \Gamma_{1}, \quad \frac{\partial z_{u}}{\partial n}+\beta z_{u} \geqslant g \quad \text { on } \Gamma_{2} \text {. }
\end{aligned}
$$

We then have

Lemma 5.1. Suppose there exists a function $w>0$ on $\Omega \cup \partial \Omega\left(\partial \Omega=\Gamma_{1} \cup \Gamma_{2}\right)$ such that

$$
\left(\nabla^{2}+h\right) w \leqslant 0 \quad \text { on } \Omega, \quad \frac{\partial w}{\partial n}+\beta w \geqslant 0 \quad \text { on } \Gamma_{2}
$$

Then, for any lower solution $z_{l}$ of (21) the inequality $z_{l} \leqslant z$ holds. The converse is also true for upper solutions, i.e. $z \leqslant z_{u}$ for any upper solution. If $h \leqslant 0$ and $\beta \geqslant 0$ then $w \equiv 1$ is sufficient.

For a proof see [24, Chapter 2, Section 6], and the following remark:

Remark 5.1. The proof of Lemma 5.1 is based on showing the nonpositivity, respectively the nonnegativity, of the functions $\left(z-V_{u}\right) / w$ and $\left(V_{l}-z\right) / w$ where $V_{u}$ and $V_{l}$ are upper and lower solutions defined by (22). The result remains true for problems having $\beta=0$ and $h=0$, provided $\Gamma_{2} \neq \emptyset$. Lower and upper solutions are defined with these equalities in (22). Also, in the present problem $w \equiv 1$ will suffice since $\beta \geqslant 0$ ( $\beta=0$ for the temperature equation) and $\omega \geqslant 0$. Therefore we can always guarantee the existence of a function $w$ in Lemma 5.1. The relationship between problem (21) and the system of Eqs. (13) and (14) will become clear in the proof of Theorem 5.1, which now follows.

Proof of Theorem 5.1. The assumption that $\Omega$, namely satisfies the interior sphere condition ensures that the classical maximum principles (see [24] or [13]) are applicable. 
For any solution $(u, v) \in C^{2}(\Omega) \cap C(\bar{\Omega}) \times C^{2}(\Omega) \cap C(\bar{\Omega}), \omega(u)>0$ and we write the second of Eqs. (9) as

$$
\left(\nabla^{2}-\frac{\omega}{\varepsilon}\right) v=0 \text { on } \Omega,\left(\frac{\partial v}{\partial n}+\beta v\right)=0 \text { on } \Gamma_{2}, v=v_{0}>0 \quad \text { on } \Gamma_{1} .
$$

By Remark 5.1 we choose $w \equiv 1$, i.e. $w$ satisfies $\left(\nabla^{2}+\omega / \varepsilon\right) w \leqslant 0$ on $\Omega$ and $\partial w / \partial n+$ $\beta w \geqslant 0$ on $\Gamma_{1}$. Clearly $v_{l}=0$ is a lower solution satisfying (22) and $v_{u}=\sup _{x \in \Gamma_{1}} v_{0}(x)$ is an upper solution, so that by the comparison Lemma 5.1, a classical solution $v$ satisfies $0 \leqslant v \leqslant \bar{v}$ where $\bar{v}=\sup v_{0}$. Having shown that $v \geqslant 0$ we now know that

$$
\nabla^{2} u=-Q \omega v \leqslant 0 \quad \text { on } \Omega, \quad \frac{\partial u}{\partial n}=\alpha>0 \quad \text { on } \Gamma_{2}, \quad u=u_{0} \quad \text { on } \Gamma_{1} .
$$

The weak maximum principle then asserts that the minimum is achieved on the boundary $\partial \Omega$. By the boundary point (Hopf's) lemma and the fact that $\partial u /\left.\partial n\right|_{\Gamma_{2}}>0$, the minimum cannot be attained on $\Gamma_{2}$. Thus, $u \geqslant \underline{u}:=\inf _{x \in \Gamma_{2}} u_{0}$. From assumption (6) that $\omega \leqslant \omega_{u}$ and from the previous result that $v \leqslant \bar{v}$, we see that the solution of the problem

$$
\nabla^{2} u_{u}=-Q \omega_{u} \bar{v}<\infty \text { on } \Omega, \quad \frac{\partial u_{u}}{\partial n}=\alpha>0 \text { on } \Gamma_{2}, \quad u_{u}=u_{0} \text { on } \Gamma_{1} .
$$

is an upper solution of (25) in the sense of Remark 5.1. We label the solution of problem (26) $\bar{u}$, and obtain from Lemma 5.1 that $u \leqslant \bar{u}$. This completes the proof.

\section{Existence for Type I-full reactant consumption}

We now demonstrate the existence of solutions to problem (9)-(10). Throughout, let the assumptions (BA) regarding $\Omega$ and $\partial \Omega$ hold. It will be necessary to extend the solution operator $K$, defined by (17), to one which is completely continuous. (In all that follows any extensions of $K$ will also be labelled $K$ ). We construct such an extension by generalising $K$ to a Sobolev space and employing imbedding properties.

Consider the linear problem

$$
-\nabla^{2} h=f \quad \text { on } \Omega, \quad h=g_{1} \quad \text { on } \Gamma_{1}, \quad \frac{\partial h}{\partial n}+\beta h=g_{2} \quad \text { on } \Gamma_{2},
$$

with $f \in L^{2}(\Omega), g_{1} \in W^{3 / 2,2}\left(\Gamma_{1}\right), g_{2} \in W^{1 / 2,2}\left(\Gamma_{2}\right)$ and $\beta \in L^{\infty}\left(\Gamma_{2}\right)$. Because $\Gamma_{i}, i=1,2$, are Lipschitz, the trace operator $\gamma_{0}: W^{l, 2}(\Omega) \rightarrow W^{l-(1 / 2), 2}(\Gamma):=W^{l-(1 / 2), 2}\left(\Gamma_{1}\right) \times$ $W^{l-(1 / 2), 2}\left(\Gamma_{2}\right)$ is continuous and has a continuous right inverse $\varepsilon: W^{l-(1 / 2), 2}(\Gamma) \rightarrow$ $W^{l, 2}(\Omega)$, i.e. $\gamma_{0}(\varepsilon \phi)=\phi$. Define

$$
\tilde{g}_{1}= \begin{cases}g_{1}, & x \in \Gamma_{1}, \\ 0, & x \in \Gamma_{2},\end{cases}
$$

then $\tilde{g}_{1} \in W^{3 / 2,2}(\Gamma)$ and we can extend it to the whole of $\Omega$ by $\mathscr{E} \tilde{g}_{1} \in W^{2,2}(\Omega)$. We now form the variational problem associated with BVP (27). Thus, we seek

$$
w:=h-\mathscr{E} \tilde{g}_{1} \in V:=\left\{\psi \in W^{1,2}(\Omega):\left.\gamma_{0} \psi\right|_{\Gamma_{1}}=0\right\}
$$


such that for all $\phi \in V$

$$
\begin{aligned}
& A(w, \phi):=\int_{\Omega} \nabla \phi \cdot \nabla w+\int_{\Gamma_{2}} \beta\left(\gamma_{0} w\right)\left(\gamma_{0} \phi\right)=F(\phi), \\
& F(\phi):=-\left(\phi, f-\nabla^{2} \mathscr{E} \tilde{g}_{1}\right)+\int_{\Gamma_{2}} g_{2}\left(\gamma_{0} \phi\right) .
\end{aligned}
$$

Note that $V$ is a closed subspace of $W^{1,2}(\Omega)$, in fact $W_{0}^{1,2}(\Omega) \subset V \subset W^{1,2}(\Omega)$. Denoting the dual space of $V$ by $V^{\prime}$ we form the Gelfand triple

$$
V \hookrightarrow L^{2}(\Omega) \hookrightarrow V^{\prime},
$$

where the second imbedding is compact. Since $L^{2}(\Omega) \subset V^{\prime}, f \in V^{\prime}$-remember that by the definition and properties of a Gelfand triple, the image of the second imbedding is dense in $V^{\prime}$ (with respect to the functional norm $\|\cdot\|_{V^{\prime}}$ ), and therefore it is possible to approximate each functional in $V^{\prime}$ by the means of the $L^{2}(\Omega)$-inner product. It is easy to show that the bilinear form $A(\cdot, \cdot): V \times V \rightarrow \mathbb{R}$ is $V$-elliptic (this follows from the $V$-ellipticity of its principal part) and that it is bounded below:

$$
|A(u, u)| \geqslant\|u\|_{1,2}^{2} \quad \text { and } \quad|A(u, \phi)| \leqslant\|u\|_{1,2}\|\phi\|_{1,2}, \forall u, \phi \in V,
$$

respectively. The functional $F$ in (28) is bounded for all $\phi \in V$ and satisfies

$$
\|F\|_{V^{\prime}} \leqslant C\left(\|f\|+\left\|g_{1}\right\|_{3 / 2,2}^{\Gamma_{1}}+\left\|g_{2}\right\|_{1 / 2,2}^{\Gamma_{2}}\right),
$$

Thus, from the theory of strongly elliptic equations, we can conclude the following about problem (28):

Lemma 6.1. Let the assumptions made above hold. In addition, assume that the surface measure of $\Gamma_{1}$ on $\partial \Omega$ is nonzero. Then

- Problem (27) possesses a unique solution $h=g+w$, where $w \in V$ for $f \in L^{2}(\Omega)$ (Lax-Milgram).

- For some $C>0$ the unique solution satisfies the estimate

$$
\|h\|_{1,2}^{\Omega} \leqslant C\left(\|f\|^{\Omega}+\left\|g_{1}\right\|_{3 / 2,2}^{\Gamma_{1}}+\left\|g_{2}\right\|_{1 / 2,2}^{\Gamma_{2}}\right) .
$$

- The solution is in the class $W^{2,2}(\Omega)$ and satisfies

$$
\|h\|_{2,2}^{\Omega} \leqslant C\left(\|u\|_{1,2}^{\Omega}+\|f\|^{\Omega}+\left\|g_{1}\right\|_{3 / 2,2}^{\Gamma_{1}}+\left\|g_{2}\right\|_{1 / 2,2}^{\Gamma_{2}}\right) .
$$

We provide a sketch of the proof of Lemma 6.1 in the Appendix (Lemma A.1). Following the remarks at the beginning of this section we now prove the following, based on the results above:

Theorem 6.1. The operator $K: C_{a}(\bar{\Omega}) \times C_{a}(\bar{\Omega}) \rightarrow C_{2, a b}(\bar{\Omega}) \times C_{2, a b}(\bar{\Omega})$ defined by (17) can be extended to an operator $K: C(\bar{\Omega}) \times C(\bar{\Omega}) \rightarrow C_{\eta}(\bar{\Omega}) \times C_{\eta}(\bar{\Omega}), 0 \leqslant \eta<\frac{1}{2}$, which is continuous and compact. 
Proof. Since the system of Eqs. (11) is uncoupled, from Lemma 6.1 we have that, for $\hat{\sim} \in C(\bar{\Omega}) \times C(\bar{\Omega})$

$$
\|\underset{\sim}{u}\|_{W^{2,2}(\Omega)^{2}} \preccurlyeq C\left(\|R \underset{\sim}{\sim}\|_{L^{2}(\Omega)^{2}}+\left\|G_{1}\right\|_{W^{3 / 2,2}\left(\Gamma_{1}\right)^{2}}+\left\|G_{2}\right\|_{W^{1 / 2,2}\left(\Gamma_{2}\right)^{2}}\right) .
$$

Thus, the operator $K$ can be extended to $K: C(\bar{\Omega}) \times C(\bar{\Omega}) \rightarrow W^{2,2}(\Omega) \times W^{2,2}(\Omega)$ and satisfies

$$
\|K \underset{\sim}{\hat{u}}\|_{W^{2,2}(\Omega)^{2}} \preccurlyeq C|\Omega \| R \underset{\sim}{\hat{u}}|_{C(\bar{\Omega})^{2}}+c\left(G_{1}, G 2\right)
$$

for positive constants $C$ and $c$. Let us demonstrate that this extension $K$ is continuous. Let $\underset{\sim}{\sim}{ }_{1} \in C(\bar{\Omega}) \times C(\bar{\Omega})$ and $\underset{\sim}{u_{2}} \in C(\bar{\Omega}) \times C(\bar{\Omega})$ be such that $\left|\underset{\sim}{u_{1}}-{\underset{\sim}{u}}_{2}\right|_{C(\bar{\Omega})^{2}} \prec 1$. We have, from (11), that

$$
\begin{array}{ll}
E\left(K{\underset{\sim}{u}}_{1}-K{\underset{\sim}{u}}_{2}\right)=R{\underset{\sim}{u}}_{2}-R \sim_{\sim}^{u} & \text { on } \Omega, \\
B\left(K{\underset{\sim}{u}}_{1}-K{\underset{\sim}{u}}_{2}\right)=\varnothing \quad \text { on } \Gamma_{2}, \quad I_{2}\left(K \underset{\sim}{u_{1}}-K \underset{\sim}{u_{2}}\right)=\varnothing \quad \text { on } \Gamma_{2},
\end{array}
$$

so that by (33), $K{\underset{\sim}{u}}_{1}-K{\underset{\sim}{u}}_{2}$ satisfies

$$
\left\|K{\underset{\sim}{u}}_{1}-K{\underset{\sim}{u}}_{2}\right\|_{W^{2,2}(\Omega)^{2}} \preccurlyeq C\left|F{\underset{\sim}{u}}_{1}-F{\underset{\sim}{u}}_{2}\right|_{C(\bar{\Omega})^{2}},
$$

which by the continuity of $F: C(\bar{\Omega}) \times C(\bar{\Omega}) \rightarrow C(\bar{\Omega}) \times C(\bar{\Omega})$ means that $K$ is continuous. Now, by the relations

$$
W^{2,2}(\Omega) \underset{\operatorname{comp}}{\rightarrow} C_{\eta}(\bar{\Omega}) \underset{\operatorname{comp}}{\rightarrow} C(\bar{\Omega}),
$$

where $\eta<\frac{1}{2}$, we have the complete continuity of the extension of $K$ to $K: C(\bar{\Omega}) \times$ $(\bar{\Omega}) \rightarrow C_{\eta}(\bar{\Omega}) \times C_{\eta}(\bar{\Omega})$.

Lemma 6.2. Let $K: C(\bar{\Omega}) \times(\bar{\Omega}) \rightarrow C(\bar{\Omega}) \times C(\bar{\Omega})$ be the completely continuous operator constructed in the previous theorem. For any $u \in C(\bar{\Omega}) \times C(\bar{\Omega})$ and $\lambda \in[0,1]$, $\underset{\sim}{u}=\lambda K \underset{\sim}{u}$ if and only if $(u, v)$ is a solution of $(13)-(14)$ in the class $C_{2, b \eta}(\bar{\Omega}) \times C_{2, b \eta}(\bar{\Omega})$, where $\eta \in\left[0, \frac{1}{2}\right)$.

Proof. Suppose that $\underset{\sim}{u} \in C(\bar{\Omega}) \times C(\bar{\Omega}), \lambda \in[0,1]$ and $\underset{\sim}{u}=\lambda K \underset{\sim}{u}$. Because $\underset{\sim}{u} \in C(\bar{\Omega}) \times C(\bar{\Omega})$, by the extension constructed in Theorem 6.1, $\lambda \underset{\sim}{\sim} \underset{\sim}{\sim} \in C_{\eta}(\bar{\Omega}) \times C_{\eta}(\tilde{\bar{\Omega}})$ for $\eta \in\left[0, \frac{1}{2}\right)$

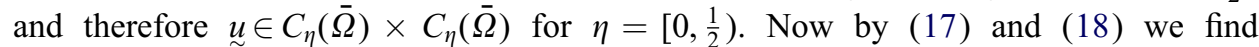
that $\lambda K \underset{\sim}{\sim} \in C_{2, b \eta}(\bar{\Omega}) \times C_{2, b \eta}(\bar{\Omega})$ for $\eta \in\left[0, \frac{1}{2}\right)$ and therefore $\underset{\sim}{u} \in C_{2, b \eta}(\bar{\Omega}) \times C_{2, b \eta}(\bar{\Omega})$ for $n \in\left[0, \frac{1}{2}\right)$.

On the other hand, suppose that $(u, v) \in C_{2, b \eta}(\bar{\Omega}) \times C_{2, b \eta}(\bar{\Omega})$. Then the converse follows trivially from definition (17) of $K$.

We now come to the main result concerning the full ignition problem

Theorem 6.2. Let the assumptions $(B A)$ regarding the boundary be satisfied. Let $g_{1} \in C_{2, b}\left(\Gamma_{1}\right) \cap W^{3 / 2,2}\left(\Gamma_{1}\right), g_{2} \in C_{1, b}\left(\Gamma_{2}\right) \cap W^{1 / 2,2}\left(\Gamma_{2}\right), f \in C_{b}(\bar{\Omega}) \cap W^{1,2}(\Omega)$ and $\beta \in$ $W^{1, \infty}\left(\Gamma_{2}\right) \cap C_{1, b}\left(\Gamma_{2}\right)$. Then there exists at least one solution to the steady state of system (4)-(5) in $C_{2, b \eta}(\bar{\Omega}) \times C_{2, b \eta}(\bar{\Omega}), \eta \in\left[0, \frac{1}{2}\right)$. 
Proof. Let $K: C(\bar{\Omega}) \times C(\bar{\Omega}) \rightarrow C(\bar{\Omega}) \times C(\bar{\Omega})$ be the completely continuous operator constructed above and substitute it into $(15)$ with $\mathscr{S}=C(\bar{\Omega})$. We have constructed a homotopy of fixed points of the map $u=\lambda K \hat{u}, \lambda \in[0,1]$, where for each $\lambda$, the fixed point is a solution of system (13) and (14) in $C_{2, b \eta}(\bar{\Omega}) \times C_{2, b \eta}(\bar{\Omega}), \eta \in\left[0, \frac{1}{2}\right)$, by virtue of the preceding lemma. Now let

$$
\begin{aligned}
& \underset{\sim}{\bar{u}}:=(\bar{u}, \bar{v})^{\mathrm{T}}, \\
& \Sigma:=\left\{\underset{\sim}{u} \in C(\bar{\Omega}) \times C(\bar{\Omega}):|\underset{\sim}{u}|_{C(\bar{\Omega})^{2}} \prec \underset{\sim}{\bar{u}}+\delta, \underset{\sim}{u} \neq \varnothing\right\},
\end{aligned}
$$

where $\mathbb{R} \times \mathbb{R} \ni \delta \succ \varnothing$ and $\bar{v}, \underline{u}$ and $\bar{u}$ are defined in Theorem 5.1. From Theorem 5.1 the set of solutions ${\underset{\sim}{u}}_{\lambda}$ of $\underset{\sim}{u}=\lambda K \underset{\sim}{u}$ have the property that $\left\{{\underset{\sim}{u}}_{\lambda}\right\} \subset$ int $\Sigma$, with strict inclusion, and since $K$ is completely continuous, so is $H(\cdot, \lambda)=I-\lambda K$ for each $\lambda \in[0,1]$. It follows therefore that $\operatorname{deg}(H(\cdot, \lambda), \Sigma, \varnothing)$ is defined, i.e. $\varnothing \notin H(\cdot, \lambda)(\partial \Sigma)$ for each $\lambda \in[0,1]$. To sec this, suppose first that $\lambda=0$. Then $\underset{\sim}{u}=\varnothing \notin \partial \Sigma$. If $\lambda \neq 0$ then $\underset{\sim}{u}=\lambda K \underset{\sim}{u}$ is a solution of (13)-(14) and $\underset{\sim}{u} \in$ int $\Sigma$. Thus $H(\cdot, \lambda) \neq \varnothing$ for all $\underset{\sim}{u} \in \partial \Sigma$. By Homotopy Invariance, Lemma 4.1

$$
\operatorname{deg}(H(\cdot, \lambda), \Sigma, \varnothing)=\operatorname{deg}(H(\cdot, 0), \Sigma, \varnothing)=1, \quad \forall \lambda \in(0,1]
$$

Therefore, since the degree is nonzero for $\lambda=1$, the equation $\underset{\sim}{u}=K \underset{\sim}{u}$ has at least one solution $\underset{\sim}{u} \in \Sigma$, which satisfies the bound (18).

Notice that a-priori boundedness of all classical steady-states is a sufficient requirement for existence. From Section 5 we know that such solutions are always bounded. This follows directly from the (strongly) sublinear behaviour of $\omega(s)$ as $s \rightarrow \infty$.

\section{Existence and nonexistence for Type II-small reactant consumption and weak power-sources}

For many bulk materials such as moist wood chips, wool, compost, ammonium nitrate and lead azide, the approximation of no-reactant-consumption is a valid one since these systems vary little from their initial state. In such cases, we can go even further and introduce the so-called large activation energy limit $E_{\mathrm{a}} \rightarrow \infty$, which leads naturally to a Frank-Kamenetskii type problem [12] (what we have called type II) for the temperature perturbation due to self heating. An interesting limit in the context of induced burning is then that of a small power source, and since we are employing the large activation energy limit, it would be natural to consider $\alpha$ to be $O\left(1 / E_{\mathrm{a}}\right)$.

For the systems described above it is realistic to take $\hat{T}_{0}$ and $\hat{X}_{0}$ as uniform. We can nondimensionalize essentially as before but with $T=u \hat{T}_{0}$ defining the temperature $u$. Let $\delta=R \hat{T}_{0} / E_{\mathrm{a}}$, and note that $\delta$ is a small parameter in the large activation-energy limit. Expanding $u, v$ and $\alpha$ as

$$
u \sim 1+\delta \theta, \quad v \sim 1-\delta \psi, \quad \alpha=O(\delta)
$$


we obtain the following leading-order problem for the temperature perturbation $\theta$

$$
\theta_{t}-\nabla^{2} \theta=Q \Lambda e^{\theta} \quad \text { on } \Omega, \quad \frac{\partial \theta}{\partial n}=\alpha \quad \text { on } \Gamma_{2}, \quad \theta=0 \quad \text { on } \Gamma_{1} .
$$

$\Lambda$ is a reduced Damköhler number that depends at most algebraically on $\delta$. Notice that the mass-fraction and temperature equations, as already mentioned, are decoupled at this order.

We now set about studying the existence of steady-state solutions to this problem after generalizing $e^{\theta}$ by a function $\omega(\theta)$ that satisfies properties (7) and (8).

The matter of existence of steady-state solutions to (37) reduces to finding upper and lower solutions, as has already been demonstrated in the preceding analysis. The Leray-Schauder degree approach remains essentially unchanged. That is

Lemma 7.1. If solutions $\theta \in C(\bar{\Omega}) \cap C^{2}(\Omega)$ of

$$
\nabla^{2} \theta=-\Lambda \omega(\theta) \quad \text { on } \Omega, \quad \theta=0 \quad \text { on } \Gamma_{1}, \quad \frac{\partial \theta}{\partial n}=\alpha \quad \text { on } \Gamma_{2},
$$

satisfy $\underline{\theta} \leqslant \theta \leqslant \bar{\theta}$, there exists at least one $\theta \in C_{2, a}(\Omega), a \in\left[0, \frac{1}{2}\right)$, satisfying (38).

Proof. Suppose $\hat{\theta}_{\lambda}$ is a known function. Consider the family of linear problems of finding $\theta_{\lambda}, \lambda \in[0,1]$, such that

$$
\nabla^{2} \theta_{\lambda}+Q \lambda \Lambda \omega\left(\hat{\theta}_{\lambda}\right)=0 \text { on } \Omega, \theta_{\lambda}=0 \text { on } \Gamma_{1}, \frac{\partial \theta_{\lambda}}{\partial n}=\lambda \alpha(x) \text { on } \Gamma_{2} \text {. }
$$

We can define an operator $K: C_{a}(\bar{\Omega}) \rightarrow C_{2, a}(\bar{\Omega}), \theta \mapsto \hat{\theta}_{\lambda}, a \in(0,1]$, using the procedure in Section 6 and the Appendix. Furthermore, by Theorem $6.1 \mathrm{~K}$ can be extended to $K: C(\bar{\Omega}) \rightarrow C_{a}(\bar{\Omega})$, where $a \in\left[0, \frac{1}{2}\right)$, and is completely continuous. Thus the proof of Lemma 6.2 remains valid. The only remaining requirement therefore, is that classical solutions are bounded. For then we can apply Theorem 6.2 to conclude the existence of at least one solution.

However, now we can show that in some cases steady-state solutions of the perturbation problem do not exist. The first result in this direction is as follows

Lemma 7.2. Let $\omega$ satisfy (8). BVP (37) has no nonnegative steady-state solutions for $\Lambda>\Lambda_{0}$, where $k_{2} \Lambda_{0}$ is the principal eigenvalue of

$$
\nabla^{2} \eta=-\Lambda k_{2} \eta \quad \text { on } \Omega, \quad \eta=0 \quad \text { on } \Gamma_{1}, \quad \frac{\partial \eta}{\partial n}=0 \quad \text { on } \Gamma_{2} \text {. }
$$

Proof. For $\Lambda>\Lambda_{0}$ consider the BVP

$$
\nabla^{2} \phi=-\Lambda\left(k_{1}+k_{2} \phi\right) \quad \text { on } \Omega, \quad \phi=0 \quad \text { on } \Gamma_{1}, \quad \frac{\partial \phi}{\partial n}=0 \quad \text { on } \Gamma_{2} .
$$

A lower solution $\underline{\phi}$ of this problem is defined by

$$
\nabla^{2} \underline{\phi} \geqslant-\Lambda\left(k_{1}+k_{2} \underline{\phi}\right) \quad \text { on } \Omega, \quad \underline{\phi} \leqslant 0 \quad \text { on } \Gamma_{1}, \quad \frac{\partial \underline{\phi}}{\partial n} \leqslant 0 \quad \text { on } \Gamma_{2} .
$$


An upper solution $\bar{\phi}$ is defined by reversing these inequalities and lower (upper) solutions satisfy $\phi \leqslant \phi(\bar{\phi} \geqslant \phi)$. The proof of this comparison result is the same as the proof of Lemma 5.1, with the function $w \in C^{\infty}(\tilde{\Omega})$ given by a solution of the Helmholtz problem

$$
\nabla^{2} w=-\Lambda^{*} w \quad \text { on } \tilde{\Omega}, \quad w=0 \quad \text { on } \tilde{\Gamma}_{1}, \quad \frac{\partial w}{\partial n}=0 \quad \text { on } \tilde{\Gamma}_{2}
$$

where $\Lambda^{*}>\Lambda$ and $\tilde{\Omega} \supset \supset \Omega$ is a $C^{\infty}$ region with boundary $\tilde{\Gamma}_{1} \cup \tilde{\Gamma}_{2}$. The function $w$ is positive on $\Omega$ and for $\tilde{\Omega} \in C^{m}, w \in W^{m, 2}(\tilde{\Omega})$. The existence of such a $w$ follows from Section 3.6 of [3]. The eigenfunctions $w_{j}$ of (43) form a complete orthonormal system and the corresponding sequence of eigenvalues $\left\{\lambda_{j}\right\}_{j=1}^{\infty}, \lambda_{j} \geqslant 0$, satisfies $\lim _{j \rightarrow \infty} \lambda_{j}=\infty$. Thus, we can always find a $\Lambda^{*}>\Lambda$ and a nonnegative (on $\tilde{\Omega}$ ) eigenfunction $w_{j}$ to satisfy (43).

Clearly $\underline{\phi} \equiv 0$ is a lower solution of (41), and by the relations

$$
\begin{aligned}
& \nabla^{2} \theta_{\Lambda}=-\Lambda \omega\left(\theta_{\Lambda}\right) \leqslant-\Lambda\left(k_{1}+k_{2} \theta_{\Lambda}\right) \quad \text { on } \Omega, \\
& \theta_{\Lambda}=0 \geqslant 0 \quad \text { on } \Gamma_{1}, \quad \frac{\partial \theta_{\Lambda}}{\partial n}=\alpha \geqslant 0 \quad \text { on } \Gamma_{2},
\end{aligned}
$$

we conclude that $\theta_{\Lambda}$, a steady-state solution of (37) for $\Lambda \geqslant \Lambda_{0}$, is an upper solution of (41). Thus, $0 \leqslant \phi \leqslant \theta_{\Lambda}$ and by Lemma 7.1 there exists at least one solution $\phi \in C_{2, a}(\Omega), a \in\left[0, \frac{1}{2}\right)$. The solutions $\phi$ are positive on $\Omega$ since $\nabla^{2} \phi<0$ and therefore, by the maximum principle, the minimum is achieved on the boundary. The boundary point lemma prevents a minimum being attained on $\Gamma_{2}$; thus $\phi$ is nonnegative on $\bar{\Omega}$ and is positive on $\Omega$.

Let $\eta_{0}$ be a nonnegative eigenfunction of (40) corresponding to $\lambda_{0}$. Integrating the identity $\left(\phi \nabla^{2} \eta_{0}-\eta_{0} \nabla^{2} \phi\right)$ over $\Omega$ yields

$$
\begin{aligned}
0 & =\int_{\Omega}\left(\phi \nabla^{2} \eta_{0}-\eta_{0} \nabla^{2} \phi\right) \\
& =\int_{\Omega}\left(\Lambda \eta_{0}\left(k_{1}+k_{2} \phi-\Lambda_{0} k_{2} \eta_{0} \phi\right)\right. \\
& =k_{2}\left(\Lambda-\Lambda_{0}\right) \int_{\Omega} \eta_{0} \phi+\Lambda k_{1} \int_{\Omega} \eta_{0} .
\end{aligned}
$$

This, along with the positivity of $\phi$ and $\eta_{0}$ on $\Omega$ implies that

$$
k_{2}\left(\Lambda_{0}-\Lambda\right) \int_{\Omega} \eta_{0} \phi=\Lambda k_{1} \int_{\Omega} \eta_{0}>0,
$$

which in turn implies that $\Lambda_{0}>\Lambda$, a contradiction.

Finally we can show the following:

Corollary 7.1. There exists a $\Lambda_{u} \in(0, \infty)$ such that $B V P$ (38) has a nonnegative solution for $0<\Lambda<\Lambda_{u}$ and no solution for $\Lambda \geqslant \Lambda_{u}$. 
Proof. First, we note that by the maximum and boundary-point principles solutions $\theta$ are nonnegative, provided $\Lambda>0$. Now we define an upper solution $\bar{\theta}$ of BVP (38) by

$$
\nabla^{2} \bar{\theta}+\Lambda \omega(\bar{\theta}) \leqslant 0 \quad \text { on } \Omega, \quad \bar{\theta} \geqslant 0 \quad \text { on } \Gamma_{1}, \quad \frac{\partial \bar{\theta}}{\partial n}-\alpha \geqslant 0 \quad \text { on } \Gamma_{2}
$$

and assert that $\bar{\theta} \geqslant \theta$ on $\bar{\Omega}$. To see this, set $\pi:=\bar{\theta}-\theta$ and use (38) and (47) to obtain

$$
\nabla^{2} \pi+\Lambda k \pi \leqslant 0 \quad \text { on } \Omega, \quad \pi \geqslant 0 \quad \text { on } \Gamma_{1}, \quad \frac{\partial \pi}{\partial n} \geqslant 0 \quad \text { on } \Gamma_{2},
$$

where we have used the continuity of $\omega(s)$ (with constant $k$ ). Note that $\underline{\pi} \equiv 0$ satisfies (48) with equality replacing the inequalities. Therefore, in the sense of lower and upper solutions defined by Eq. (42) (and the inequalities reversed), the function $\pi$ is nonnegative, i.e. $\pi=\bar{\theta}-\theta \geqslant 0$ and the assertion is proved. We shall define the spectrum $\Xi$ of BVP (38) as the set of all $\Lambda$ such that (38) has a nonnegative solution. To proceed with the proof, we shall need the following:

Lemma 7.3. If $\Lambda_{*} \in \Xi \cap(0, \infty)$, then $\left[0, \Lambda_{*}\right] \subset \Xi$.

Proof. Let $\theta_{*}$ be a nonnegative solution of (42) corresponding to $\Lambda_{*}$. Then

$$
\nabla^{2} \theta_{*}=-\Lambda_{*} \omega\left(\theta_{*}\right) \leqslant-\Lambda \omega\left(\theta_{*}\right) \quad \text { on } \Omega, \quad \forall \Lambda \in\left[0, \Lambda_{*}\right) .
$$

Thus, $\theta_{*}$ is an upper solution of (38) for any $\Lambda \in\left[0, \Lambda_{*}\right)$ with $\theta_{*} \geqslant \theta_{\Lambda}$, where $\theta_{\Lambda}$ is a solution of (38) corresponding to $\Lambda$. Consequently, $0 \leqslant \theta_{\Lambda} \leqslant \theta_{*}<\infty$ and therefore by Lemma 7.1, BVP (38) has a nonnegative solution for each $\Lambda \leqslant \Lambda_{*}$.

We now demonstrate that $\Xi \neq \emptyset$. Let $\bar{\theta}$ be a solution of

$$
\nabla^{2} \bar{\theta}=-1 \quad \text { on } \Omega, \quad \bar{\theta}=0 \quad \text { on } \Gamma_{1}, \quad \frac{\partial \bar{\theta}}{\partial n}=\alpha \quad \text { on } \Gamma_{2} .
$$

Select $\Lambda_{1}$ sufficiently small that $\nabla^{2} \bar{\theta} \leqslant-\Lambda_{1} \omega(\bar{\theta})$. Then $\bar{\theta}$ is an upper solution of (38) with $\Lambda=\Lambda_{1}$. By Lemma 7.1 a nonnegative solution of (38) exists and therefore $\Xi \neq \emptyset$. Furthermore, by Lemma 7.3, $\left[0, \Lambda_{1}\right] \subseteq \Xi$.

Now define $\Lambda_{u}=\sup _{\Lambda \in \Xi} \Lambda$. To complete the proof we need to show that $\Lambda_{u}<\infty$. Because of the growth condition $\omega(s)>k_{1}+k_{2} s$, we can apply Lemma 7.2 to conclude that nonnegative solution to (38) exists for each $\Lambda>\Lambda_{0}$, where $\Lambda_{0}$ is as defined in Lemma 7.2. Thus, as required, $\Lambda_{u} \leqslant \Lambda_{0}<\infty$.

\section{Concluding remarks}

For any Damköhler number, we have demonstrated the existence of steady-state solutions to a system representing the heating of a combustible material by an internal source. In contrast, steady-state solutions of the associated perturbation problem, arising from a high activation-energy asymptotics approach, exist only for Damköhler numbers below a critical value that is characterized by the principal eigenvalue of a Helmholtz problem. 
In forthcoming work we will advance this study by characterizing the steady states corresponding to spherically symmetric geometries. In particular, we will present critical values of boundary data, heat flux and heat release at which the steady-state solutions cease to behave as smooth functions of these parameters. This involves numerical computations using a variant of the AUTO package [1,2]. Finally, we are exploring the conditions for the existence and the blow-up of the full time-dependent problem (4) and (5).

\section{Acknowledgements}

The first author is grateful to the EPSRC for financial support through Research Grant GR/R 22179 and to OCIAM, Oxford, UK for supporting a short visit.

\section{Appendix A.}

Here we shall sketch a proof of estimate (16). We let assumptions (BA) regarding $\Gamma_{1}$ and $\Gamma_{2}$ be valid and consider the BVP

$$
\nabla^{2} h+f=0 \quad \text { on } \Omega, \quad h=g_{1} \quad \text { on } \Gamma_{1}, \quad \frac{\partial h}{\partial n}+\beta h=g_{2} \quad \text { on } \Gamma_{2},
$$

where $f \in C_{\alpha}(\bar{\Omega}) \cap W^{1,2}(\Omega)$ and $\beta \in W^{1, \infty}\left(\Gamma_{2}\right) \cap C_{1, \alpha}\left(\Gamma_{2}\right)$. We have

Theorem A.1. If $g_{1} \in C_{2, \alpha}\left(\Gamma_{1}\right) \cap W^{3 / 2,2}\left(\Gamma_{1}\right)$ and $g_{2} \in C_{1, \alpha}\left(\Gamma_{2}\right) \cap W^{1 / 2,2}\left(\Gamma_{2}\right)$, then problem (A.1) possess a unique solution $h \in C_{2, \alpha}(\bar{\Omega})$ satisfying the estimate

$$
|h|_{2, \alpha}^{\Omega_{*}} \leqslant C\left(|f|_{\alpha}^{\Omega}+\left|g_{1}\right|_{2, \alpha}^{\Gamma_{1}}+\left|g_{2}\right|_{1, \alpha}^{\Gamma_{2}}\right) .
$$

In order to prove Theorem A.1 we need the following:

Lemma A.1. A solution $h$ of problem (A.1) exists, is in the class $W^{2,2}(\Omega) \cap W_{\text {loc }}^{4,2}(\Omega)$ and, for some $C>0$, it satisfies

$$
\|h\|_{2,2}^{\Omega} \leqslant C\left(\|h\|_{1,2}^{\Omega}+\|f\|^{\Omega}+\left\|g_{1}\right\|_{3 / 2,2}^{\Gamma_{1}}+\left\|g_{2}\right\|_{1 / 2,2}^{\Gamma_{2}}\right) .
$$

Proof. Existence of a weak solution in the class $W^{1,2}(\Omega)$ is assured by Lemma 6.1. By the smoothness properties of the boundary components and the properties of $g_{1}$ and $g_{1}$ it is possible to show that $h \in W^{2,2}(\Omega) \cap W_{\text {loc }}^{4,2}(\Omega)$. For given that $h \in W^{1,2}(\Omega)$, in any $\Omega^{\prime} \subset \subset \Omega$ we can use a difference-quotient argument to show that the solution is in $W^{1,2}(\Omega) \cap W_{\text {loc }}^{2,2}(\Omega)$ and by iteration, that $h \in W^{1,2}(\Omega) \cap W_{\text {loc }}^{4,2}(\Omega)$ with

$$
\|h\|_{4,2}^{\Omega^{\prime}} \leqslant C\left(\|h\|_{1,2}^{\Omega}+\|f\|_{1,2}^{\Omega}\right) .
$$


For details see [13, Theorems 8.9 and 8.10]. By a change of coordinates, neighbourhoods $G_{1}$ of the boundary $\Gamma_{1}$ can be mapped to a half-space as follows: $G_{1} \cap \Omega \rightarrow$ $G_{y} \subset\left\{y: y_{n}<0\right\}$ and $G_{1} \cap \Gamma_{1} \rightarrow \Gamma_{y} \subset\left\{y: y_{n}<0\right\}$, where $y_{i}, i=1, n$ are the new coordinates. Then by a cut-off function argument (see [21, Theorem 4.18]).

$$
\|h\|_{2,2}^{\Omega \cap G_{1}^{\prime}} \leqslant C\left(\|h\|_{1,2}^{\Omega \cap G_{1}}+\|f\|^{\Omega \cap G_{1}}+\left\|g_{1}\right\|_{3 / 2,2}^{\Gamma_{1} \cap G_{1}}\right)
$$

for any $G_{1}^{\prime} \subset \subset G_{1}$. A similar, but not identical, argument (again see [21]) for neighbourhoods $G_{2}$ of $\Gamma_{2}$ leads to

$$
\|h\|_{2,2}^{\Omega \cap G_{2}^{\prime}} \leqslant C\left(\|h\|_{1,2}^{\Omega \cap G_{2}}+\|f\|^{\Omega \cap G_{2}}+\left\|g_{2}\right\|_{1 / 2,2}^{\Gamma_{2} \cap G_{2}}\right)
$$

for any $G_{2}^{\prime} \subset \subset G_{2}$. Estimates (A.4)-(A.6) and the compactness of the boundary imply that $h \in W^{2,2}(\Omega) \cap W_{\text {loc }}^{4,2}(\Omega)$, and combining them gives (A.3).

Proof of Theorem A.1. The existence of a solution in the class $C^{2}(\Omega) \cap C(\bar{\Omega})$ is assured by the previous lemma and the Sobolev Imbedding Theorem:

$$
W^{k, 2}(\Omega) \underset{\operatorname{comp}}{\rightarrow} C^{m}(\bar{\Omega}), \quad 0 \leqslant m<k-n / 2 .
$$

The proof of Theorem A.1 requires a Green's representation of the solution:

$$
h(y)=\int_{\Omega} \Lambda(x-y) \nabla^{2} h+\int_{\Gamma_{1} \cup \Gamma_{2}}\left(h \frac{\partial \Lambda}{\partial n}(x-y)-\Lambda(x-y) \frac{\partial h}{\partial n}\right),
$$

where $\Lambda$ is a fundamental solution of Laplace's equation. Since $h \in C^{2}(\Omega) \cap C(\bar{\Omega})$ and $\Lambda$ is sufficiently smooth, the integrals in this definition make sense. Based on, amongst other things, Kellogg's Theorem in a ball ([13, Corollary 6.9]), it possible to show that $h \in C_{2, \alpha}(B)$ for any ball $B \subset \subset \Omega$ (sec [13, Lemma 6.16]). This provides the necessary interior regularity. The boundary regularity follows from the smoothness of the boundary components and the boundary data. One can show that on any part $G$ of $\Gamma_{1} \in C_{2, \alpha}$, the solution is in $C_{2, \alpha}(\Omega \cup G)$ (see [13, Lemma 6.18]). Finally, regularly on the boundary component $\Gamma_{2}$ follows from Theorem 6.31 in [13].

Remark A.1. In the case of a pure Neumann condition on $\Gamma_{2}(\beta=0)$ these results remain valid with little or no modification.

\section{References}

[1] E. Balikrishnan, A. Swift, G.C. Wake, Path-following for disjoint bifurcation problems arising in ignition theory, Math. Comput. Modelling 19 (1994) 9-15.

[2] E. Balikrishnan, A. Swift, G.C. Wake, Critical values for some non-class A geometries in thermal ignition theory, Math. Comput. Modelling 24 (1996) 1-10.

[3] V. Barbu, Partial Differential Equations and Boundary Value Problems, Mathematics and its Applications, Vol. 441, Kluwer Academic Publishers, Dordrecht, 1988 (revised Edition in English). 
[4] J. Bebernes, D. Eberly, Mathematical Problems Combustion Theory, Applied Mathematical Science, Vol. 83, Springer, New York, 1989.

[5] J. Brindley, J.F. Griffiths, A.C. Mcintosh, Ignition phenomenology and criteria associated with hotspots embedded in a reactive material, Chem. Eng. Sci. 56 (2001) 2037-2046.

[6] J. Brindley, J.F. Griffiths, A.C. Mcintosh, Potential hazards from spherical hotspots in reactive solids, in: D. Bradley, D. Drysdale, G. Makhviladze (Eds.), Proceedings of the Third International Seminar on Fire and Explosion Hazards, Lake District, England 2001.

[7] J. Brindley, J.F. Griffiths, A.C. Mcintosh, J. Zhang, Initiation of combustion waves in solids and the effect of geometry, ANZIAM J. 43 (2001) 149-163.

[8] Buckmaster, Ludford, Theory of Laminar Flames, Cambridge University Press, Cambridge, 1982.

[9] J.G. Burnell, A.A. Lacey, G.C. Wake, Steady states of the reaction-diffusion equations. Part I: questions of existence and continuity of solution branches, J. Austral. Math. Soc. Ser. B 24 (1983) 374-391.

[10] J.G. Burnell, A.A. Lacey, G.C. Wake, Steady states of the reaction-diffusion equations. Part II: uniqueness of solutions and some special cases, J. Austral. Math. Soc. Ser. B 24 (1983) 392-416.

[11] J.G. Burnell, A.A. Lacey, G.C. Wake, Steady states of the reaction-diffusion equations. Part III: questions of multiplicity and uniqueness of solutions, J. Austral. Math. Soc. Ser. B 27 (1985) 88-110.

[12] D.A. Frank-Kamenetskii, Diffusion and Heat Transfer in Chemical Kinetics, Princeton University Press, Princeton, NJ, 1955.

[13] D. Gilbarg, N.S. Trudinger, Elliptic partial differential equations of second order, revised 3rd Edition, Classics in Mathematics, Springer, Berlin, 2001.

[14] B.F. Gray, W. Kordylewski, Travelling waves in exothermic systems, Proc. Roy. Soc. London A 416 (1988) 103-113.

[15] B.F. Gray, G.C. Wake, Initial conditions for thermal ignition, Math. Comput. Modelling 18 (1993) 65-75.

[16] A.K. Kapila, Evolution of a deflagration in a cold combustible subjected to a uniform energy flux, Internat. J. Eng. Sci. 19 (1981) 495-509.

[17] G.M. Lieberman, Hölder continuity of the gradient of solutions of uniformly parabolic equations with conormal boundary conditions, Ann. Matem. Pura Appl. 148 (4) (1985) 77-99.

[18] G.M. Lieberman, Mixed boundary value problems for elliptic and parabolic differential equations of second order, J. Math. Anal. Appl. 113 (1986) 422-440.

[19] A. Liñán, F.A. Williams, Theory of ignition of a reactive solid by constant energy flux, Combust. Sci. Technol. 3 (1971) 91-98.

[20] A.C. Mcintosh, J. Brindley, J.F. Griffiths, An approximate model for the ignition of reactive materials by a hot-spot with reactant depletion, in: Mathematical and Computer Modelling, 36 (2003) 293-306 (Special volume to mark the retirement of Prof. J. Brindley).

[21] W.C. Mclean, Strongly Elliptic Systems and Boundary Integral Equations, Cambridge University Press, Cambridge, 2000.

[22] G.N. Mercer, R.O. Weber, H.S. Sidhu, An oscillatory route to extinction for solid fuel combustion waves due to heat losses, Proc. Roy. Soc. London A 454 (1999) 2015-2022.

[23] G.N. Mercer, R.O. Weber, B.F. Gray, S.D. Watt, Combustion pseudowaves, Math. Comput. Modelling 24 (1996) 29-38.

[24] M.H. Protter, H.F. Weinberger, Maximum Principles in Differential Equations, 2nd Edition, Springer, Berlin, 1984.

[25] R.E. Rothe, Introduction to Various Aspects of Degree Theory in Banach Spaces, Mathematical Surveys and Monographs, Vol. 23, American Mathematical Society, Providence, RI, 1986.

[26] A.A. Shah, A.C. Mcintosh, J. Brindley, J.F. Griffiths, M. Pourkashanian, The effect of oxygen on ignition phenomena in reactive solids containing a hot spot, Combust. Theory Modelling, 7 (3) (2003) 509-523.

[27] S.D. Watt, R.O. Weber, H.S. Sidhu, G.N. Mercer, A weight-function approach for determining watershed initial conditions for combustion zones, IMA J. Appl. Math. 62 (1999) 195-206.

[28] J. Wloka, Partial Differential Equations, Cambridge University Press, Cambridge, 1987 (original in German, B.G. Teubner). 